PHYSICAL REVIEW E, VOLUME 64, 059901(E)

\title{
Erratum: Superballistic spreading of wave packets [Phys. Rev. E 64, 012301 (2001)]
}

L. Hufnagel, R. Ketzmerick, T. Kottos, and T. Geisel

(Published 9 October 2001)

DOI: 10.1103/PhysRevE.64.059901

PACS number(s): 05.60.Gg, 03.65.-w, 72.20.Dp, 99.10.+g

This paper should have appeared as a regular article in Sec. E-10, General Methods of Statistical Physics [Phys. Rev. E 64, 012301 (2001)]. A typographical error made while updating the database had the unfortunate, inadvertent impact of modifying the section of this paper. The editors greatly regret this misidentification. 